\title{
Endoscopic management of ureteral calculus in a patient with ureterosigmoidostomy diversion
}

Leonardo de Albuquerque dos Santos Abreu, Celso Lara, Marco Antonio Dionísio, Alexandre Dias Pelosi, Fátima Aparecida Ferreira Figueiredo

University Gama Filho (LASA); Hospital Federal dos Servidores do Estado (LASA); Department of Urology - State University of Rio de Janeiro (CL, MAD, FAFF); Rede D'Or (ADP, FAFF) and Inca - Instituto Nacional de Câncer (ADP), Rio de Janeiro, Brazil

\section{ABSTRACT}

Lithiasis after urinary diversion is an uncommon condition that poses therapeutic challenges. The authors report the case of a patient submitted to cystectomy and ureterosigmoidostomy 35 years ago due to bladder endometriosis. The patient presented with a ureteral stone and was treated by retrograde endoscopic extraction.

\section{ARTICLE INFO}

\section{Key words:}

Urolithiasis; Urinary Diversion; Ureteral Calculi; Endoscopy

Int Braz J Urol. 2013; 39: 593-6

Submitted for publication: February 19, 2012

Accepted after revision: May 27, 2013

\section{INTRODUCTION}

Ureterosigmoidostomy has been used as a form of urinary diversion for either benign or malignant conditions. Urinary lithiasis has been reported as ureterosigmoidostomy complication in $3-10 \%$ of the cases in recent series $(1,2)$. Bacterial colonization and diversion-associated metabolic derangements are main risk factors (3) in this situation.

Treatment of urinary stones depends on clinical, anatomic and stone factors and there are many therapeutic options that might be considered when evaluating uncommon cases. Nevertheless, the choice for the best approach depends on the availability of equipment and expertise of the surgeon.

\section{CASE REPORT}

We report the case of a 62 years old woman submitted to cystectomy and ureterosigmodostomy 35 years ago due to bladder endometriosis. Potassium citrate was used to prevent hyperchloremic metabolic acidosis. The follow-up was unremarkable until the patient's current presentation with acute right flank pain. A CT scan showed left kidney agenesis and right kidney hydronephrosis. A $2 \mathrm{~cm}$ stone with $580 \mathrm{HU}$ was located in the proximal right ureter (Figure-1). 
Figure 1 - Pre-operative CT scan.
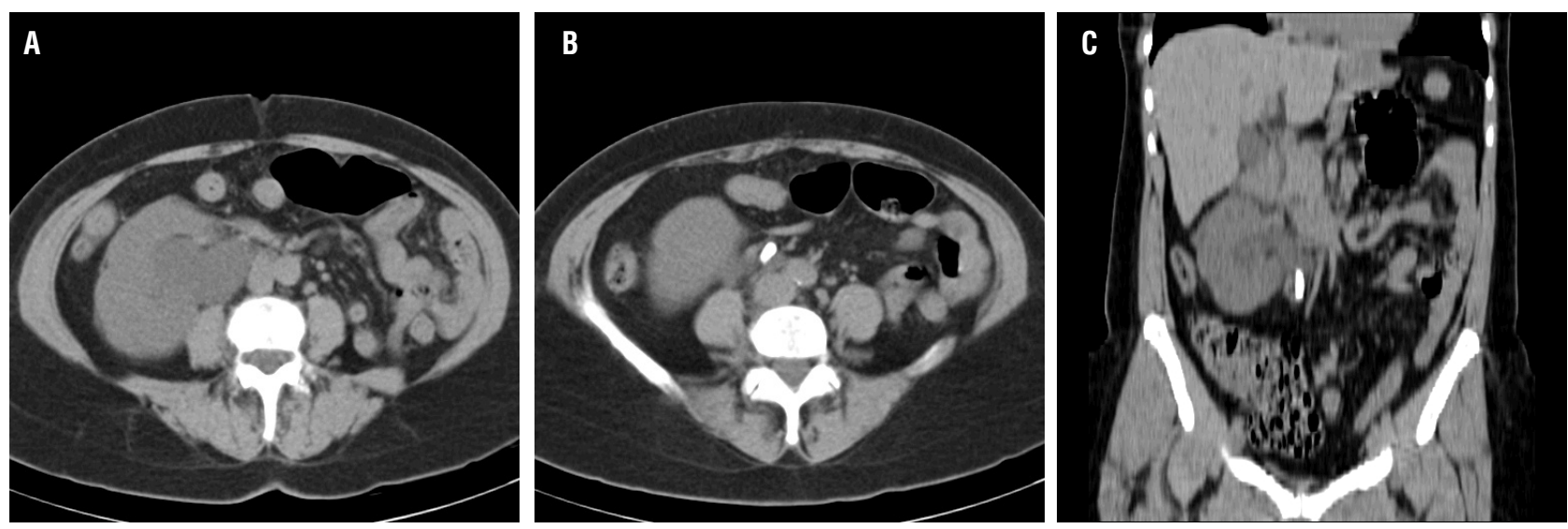

An abdominal radiograph could not show the stone confirming it as radiolucent. Serum creatinine was $1.6 \mathrm{mg} / \mathrm{dL}$. A percutaneous nephrostomy-ultrasound guided was emergently performed to relieve obstruction. While waiting for the definitive treatment the patient lost the nephrostomy and underwent a new percutaneous nephrostomy.

The initial treatment plan was to perform an anterograde holmium LASER flexible ureterolitotripsy. Under fluoroscopic guidance a middle calyx was punctured which allowed adequate access to the ureteropelvic junction (UPJ). Dilation was carried out with axial ureteral dilators over a 0.035 " guidewire and a 9.5Fr access sheath was introduced until close to the UPJ entry. When the flexible ureteroscope was introduced distal migration of the stone was noticed. Because of ureteral tortuosity we were unable to perform LASER lithotripsy and retrieve the stone (Figure-2). In this procedure a retrograde access was tried, however, we were unable to catheterize the ostium.

A retrograde approach with a side view endoscope was then carried. During sigmoidoscopy, right ureteral ostium was located at a distance of approximately $15 \mathrm{~cm}$ from the anal verge. The ureteral ostium was catheterized with a cholangiopancreatography catheter (Figure-3). A retrograde pyelogram confirmed the $2 \mathrm{~cm}$ stone in the distal ureter. A 0.035 " hydrophilic guide wire was inserted and dilation of the uretero-sigmoid junction was carried with a balloon catheter (Figure-4). After dilation it was possible to see the calculus protruding through the ostium and a biliary balloon catheter was used to pull it down (Figure-5). The
Figure 2 - Ureteroscope deflection in distal ureter.
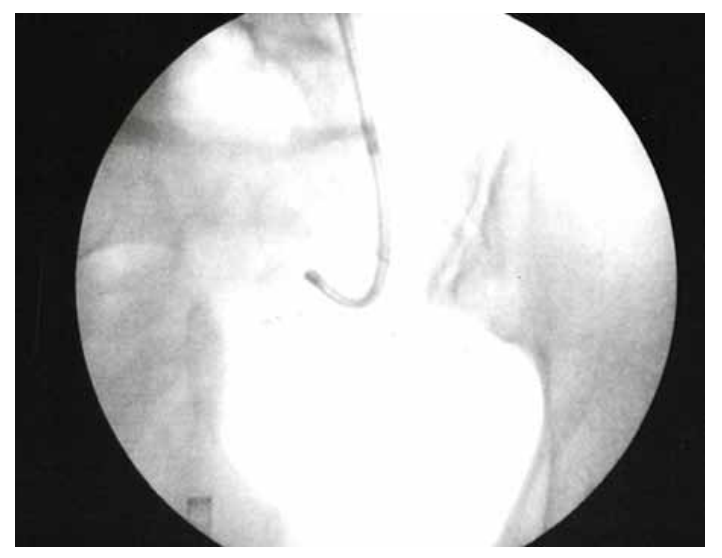

Figure 3 - Retrograde endoscopic catheterization of ureteral ostium.

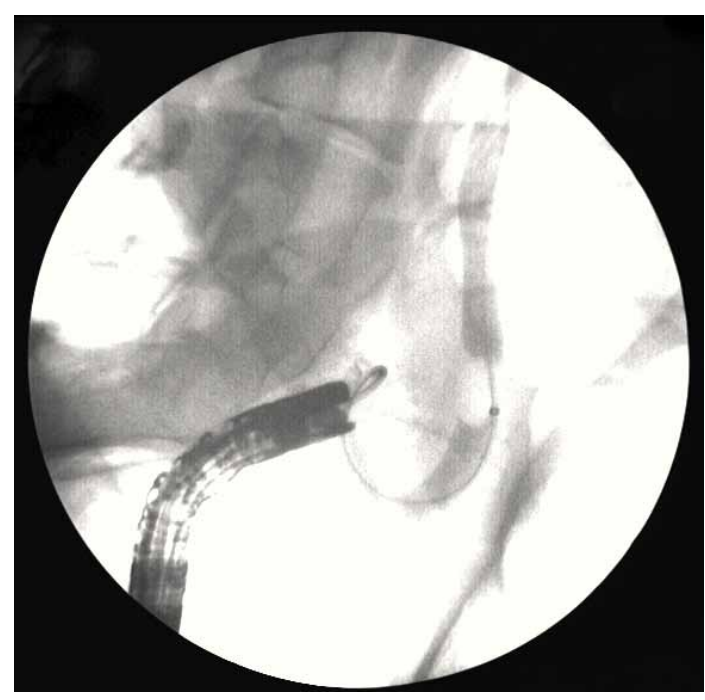


Figure 4 - Dilation of the ostium with a balloon catheter.

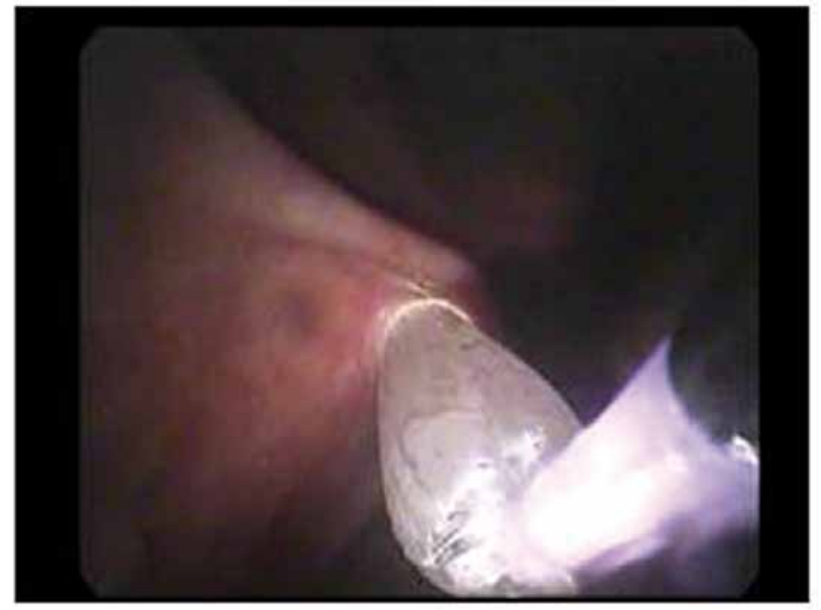

calculus had a soft appearance, however, we were unable to recover it for further composition analysis. A CT scan confirmed that the patient was stone free postoperatively and the nephrostomy was removed. Renal function returned to baseline after treatment and the patient was well until the last follow-up 1 year after the procedure.

\section{DISCUSSION}

Treatment options for ureteral stones in patients with urinary diversion range from minimally invasive procedures such as extracorporeal shock-wave lithotripsy (ESWL) to conventional open surgery.

Open surgery may be a definitive option, however, morbidity associated with a reoperation and the risks of complications must be considered. ESWL may be a good option for proximal ureteral stones but for radiolucent or distal stones the results may not be optimal $(4,5)$.

Chemolytic dissolution of stones or residual stone fragments is a useful adjunct to conventional treatment. Although uric acid stones have good response to chemolytic therapy, oral chemolysis may take several days for complete dissolution (6). Moreover, urinary alcalinization may be difficult in patients with chronic metabolic acidosis. Direct chemolysis can also be done through the nephrostomy tube. Nevertheless, the risks of infection and
Figure 5 - Extraction of the stone with the balloon catheter.

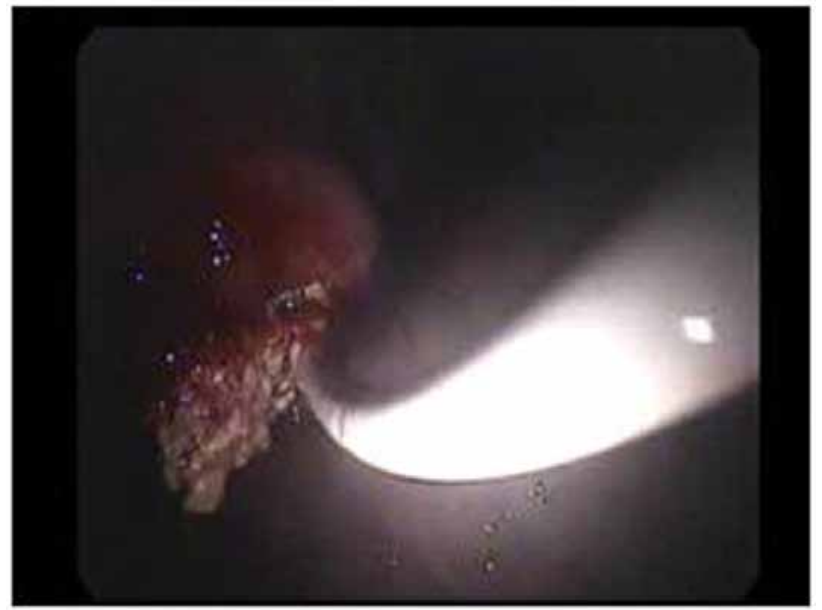

metabolic complications may be increased in this particular situation.

Choosing a retrograde or anterograde approach will depend on location of the stone. For stones located in medium or proximal ureter an anterograde approach is feasible. Renal access should be carried out in medium or upper calix to allow passage of the flexible ureteroscope without angulation as described by Lackmichi et al. (7). However, urologists must be aware that more than one flexible ureteroscope may be necessary to accomplish the procedure as was emphasized by the authors. In the reported case anterograde ureterolithotripsy was unsuccessfully because of distal migration of the stone and tortuosity of the ureter.

For stones located in the distal ureter retrograde approach may be necessary. Fitzgerald et al. (8) described ureterolithotripsy with a semirigid ureteroscope of a large ureteral stone. A guide wire was passed through a previously placed nephrostomy down the sigmoid/rectum which helped to locate and dilate the ureterointestinal junction. Mosler et al. (9) were the first to report the use of a side view duodenoscope for retrograde approach. Insufflation with air during endoscopy helped to locate the ureterointestinal junction allowing dilation of the anastomosis and removal of calculus with basket without lithotripsy. Hyams et al. (10) reported successful retrograde access in 21 of 28 attempts without the aid of previous renal 
percutaneous access. A flexible ureteroscope was also used to retrieve ureteral stones. In the reported case a side view duodenoscope was used for retrograde access and we also found that air insufflation helped location and dilation of the ureterointestinal junction. Lesion of ureterosigmoid junction during dilation for retrograde access might be of concern. Nevertheless, complications were not reported on Hyams' series, as well as in our case.

In the reported case lithotripsy was unnecessary. Nevertheless, flexible or semirigid ureteroscopes with the aid of LASER or other lithotripters may be used whenever necessary.

\section{CONCLUSIONS}

Endoscopic management of ureteral stones after urinary diversion is feasible and can be achieved with little morbidity. The use of retrograde or anterograde approach for ureteral stones after urinary diversions should combine endoscopic and endourologic technics in a multidisciplinary environment.

\section{CONFLICT OF INTEREST}

None declared.

\section{REFERENCES}

1. Tollefson MK, Elliott DS, Zincke H, Frank I: Long-term outcome of ureterosigmoidostomy: an analysis of patients with \&gt;10 years of follow-up. BJU Int. 2010; 105: 860-3.
2. Nitkunan T, Leaver R, Patel HR, Woodhouse CR: Modified ureterosigmoidostomy (Mainz II): a long-term follow-up. BJU Int. 2004; 93: 1043-7.

3. Okhunov Z, Duty B, Smith AD, Okeke Z: Management of urolithiasis in patients after urinary diversions. BJU Int. 2011; 108: 330-6.

4. Maker V, Layke J: Gastrointestinal injury secondary to extracorporeal shock wave lithotripsy: a review of the literature since its inception. J Am Coll Surg. 2004; 198: 128-35.

5. Cardinaux C, Tawadros T, Praz V, Wisard M, Treuthardt C, Jichlinski P: Ischemic cecal perforation secondary to ESWL of junctional stone in ureterosigmoidostomy (Mainz Pouch II). Urology. 2009; 73: 1423.e1-2.

6. Bernardo NO, Smith AD: Chemolysis of urinary calculi. Urol Clin North Am. 2000; 27: 355-65.

7. Lackmichi MA, Niang L, Labou I, Thibault F, Ravery V, Gattegno $B$, et al.: Anterograde flexible ureteroscopy for stones of the uretero-sigmoid junction of a Mainz II pouch. Prog Urol. 2006; 16: 505-7.

8. Fitzgerald KB, Aslan P, Preminger GM: Endourological management of a large distal ureteral calculus in a patient with ureterosigmoidostomy diversion. J Urol. 1998; 159: 2081-2.

9. Mosler P, Kiesslich R, Stein R, Galle PR, Thüroff JW, Kanzler $S$ : Use of a duodenoscope in the management of a ureteral calculus in patient with ureterosigmoidostomy (Mainz pouch II;rectosigmoid pouch). Endoscopy. 2003; 35: 1086-7.

10. Hyams ES, Winer AG, Shah 0: Retrograde ureteral and renal access in patients with urinary diversion. Urology. 2009; 74: 47-50.

\section{Correspondence address:}

Dr. Leonardo de Albuquerque dos Santos Abreu Hospital Federal dos Servidores do Estado R. Sacadura Cabral, 178 - Saúde Rio de Janeiro, RJ, 20081-262, Brazil E-mail: leonardo.abreu@sbu.org.br 